\title{
THE INFLUENCE OF SOLAR ACTIVITY ON THE PROBABILITY OF OCCURRENCE OF OCEANIC THUNDERSTORMS
}

\author{
Stuart Gathman \\ Nava1 Research Laboratory \\ Washington, DC 20375
}

\begin{abstract}
A large data base of oceanic thunderstorms was investigated for correlations with solar events. The analysis shows that there is a secondary modification of the probability of these storms in the first and second week following certain types of solar events.
\end{abstract}

\section{Introduction}

The subject of minute changes in solar emanations being the cause of tropospheric weather variations is still a cause of great controversy。 It is an area which has the future promise of significantly extending the range of long-term weather forecasts. It has been hypothesized that a physical coupling of solar events with electrical characteristics of the atmosphere may be the key in linking events on the sun with terrestrial weather patterns. In particular electrical processes may be modulated by products of solar events and thus cause a trigger of weather variations。

Recent research shows some evidence of a connecting link between solar events and the atmospheric electricity as evidence in land based thunderstorm activity (Markson, 1978; Lethbridge, 1981). However in any discussion of world-wide thunderstorm activity one must look at both 1 and-based and oceanic thunderstorms. This letter will look at solar influences on oceanic thunderstorms 。

\section{Observations}

The data base used by Trent and Gathman (1972) consisted of over 7 million meteorological observations made by ships at sea from January 1949 through June 1963. When any of the meteorological observations indicated some form of electrical activity, these records were put into a separate subfile. For each 10 by 10 degree square of the ocean, the probability of observing a thunderstorm from the deck of a ship could be obtained for that geographical area by dividing the number of records indicating electrical activity in that square by the total number of observations made in that square. This data set is a good representation of a continuous Northern Hemisphere monitor of oceanic thunderstorms. The subset of the total marine data which contains the oceanic thunderstorm observations is the data set from which the analysis in this letter is based. As each unit of the subset contains time, data and location information, a time series was made of this deck with a slot for each day throughout the entire 13 year period being filled with the number of thunderstorm observations made on that day by various ships at sea. This time series can further be modified by looking only at certain geographical areas. The time series of thunderstorm observations made in this way are not true probabilities but are related to the probabilities by the multiplication of a function whose characteristics we as sume are not solar event related.

Even though the basic marine deck used contains many observations, there are only about 1400 per day. Since it requires on the average 100 ship observations to find one with a thunderstorm, there are parts of the world wide 


\section{S. Gathman}

data base which cannot be used. The analysis of this paper is limited to the latitude belt of 0 to 35 degrees North where the results are statistically significant because of the numerous shipping lanes and high thunderstorm probabilities.

\section{Results and Discussion}

In testing this data set for possible solar event relationships the powerful method of superposed epoch analysis was used. This technique although relatively unknown is a powerful tool when properly used (Haurwitz and Brier, 1981). This technique fills a row - column array with subsets of the time series data aligned such that one particular column contains the "key" date data. The number of rows corresponds to the number of key date events contained in the time series. Column averages are then the "superposed epoch analysis". In this method a set of key dates related specifically to observed events such as the day of the first observation of a solar flare is selected. In order to be used for this analysis, each key date set had to have at least 40 days in common with the oceanic thunderstorm data set. One must however ask the following question. How significant are the variations in the value of these colum averages? For each of the 31 days. about the keydate, a collection of $\mathrm{N}$ data points exist whose average value and standard deviation can be calculated. These data may be used to test for the hypothesis that the observed deviation is significant. In the data presented below, the values of the averages for each of the 31 columns are tested for significant deviations from the combined average of the first 10 columns. This hypothesis is designed to look at changes in the oceanic thunderstorm activity which may follow the key date index. The results are plotted as a probability function. As a test of this process following the suggestions of Haurwitz and Brier (1981), several completely random keydate files were constructed and used as a control for the interpretation of the significance of the probability plots.

When the question is asked, "What is the probability that this observed deviation is the product of chance from random variations?", it is found that a value of $P$ equal to .01 means that in only 1 time out of 100 calculations with random data, would deviations of this magnitude occur.

The probability plot of a set of randomly selected key date indices is shown in Fig. 1. As expected there is no spectacular deviations throughout the 31 day period. Similar curves are obtained for all of the other random key date sets used. These plots say that all of the deviations in the superposed epoch analysis for the random key dates have a high probability of being the result of random variations in the data. Only if the probability plots drop significantly lower than . 1 (as will be shown below) is there reason to believe that the deviations in the superposed epoch analysis are significant.

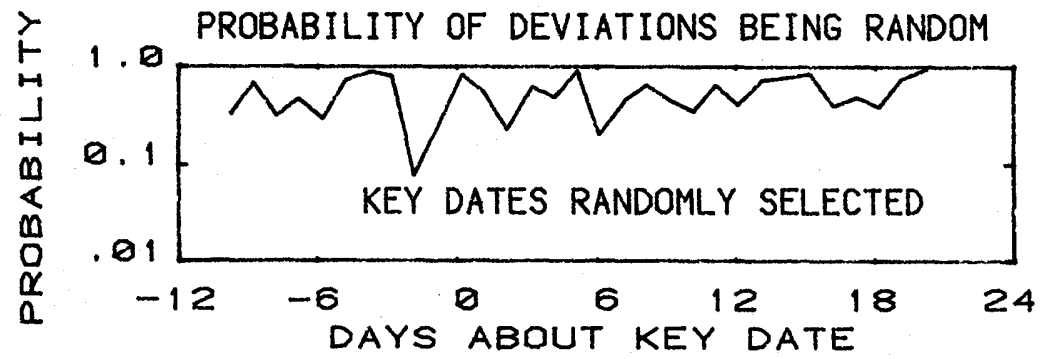

\section{FIg. I KEY DATE ANALYSIS OF OCEANIC THUNDER- STORMS IN THE LATITUDE BELT OF O TO 35 DEGREES NORTH FOR A RANDOM SET OF KEY DATES.}


Several sets of key date indices associated by various investigators with certain aspects of solar event phenomena were used with the superposed epoch analysis in conjunction with the oceanic thunderstorm time series. Geomagnetic disturbances indicated by lis.ts of geomagnetically disturbed days failed to provide any significant indications of perturbations in the oceanic thunderstorm time series. Solar rotational parameters such as the solar rotational key dates and the dates of solar sector boundary crossings (of both polarities) also failed to give any positive indications. These non-significant determinations do not necessarily mean that there is no effect (a negative is almost impossible to prove) but it does mean that at least this data base is insufficient to make a positive ascertainment as to any form of relationship between magnetic storms and solar rotational parameters and the probability of observing oceanic thunderstorms.

The positive aspects of this effort are seen by looking at the results from the key dates of charged solar particle events. The curves shown in Fig. 2 show the probability plots for three sets of key dates involving some form of solar flare indications. These data sets are:

(1) The dates of isolated solar flares (Schuurmans, 1969).

(2) The dates of type IV proton solar flare proton events (Zerofos, 1975)。

(3) The dates of significant solar proton events (Shea and Smart, 1977).

Each of these data sets is associated with charged particle events at the polar caps. The curves show that the oceanic thunderstorm time series has significant perturbations following one of these events after a sizable delay. The values of the superposed epoch analysis for each of these three sets of key dates show a decrease in oceanic thunderstorm occurrence in the second and third weeks after the key dates. These curves are different from those of Markson (1978) and Lethbridge (1981) and probably reflect differences in oceanic and land based thunderstorms 。

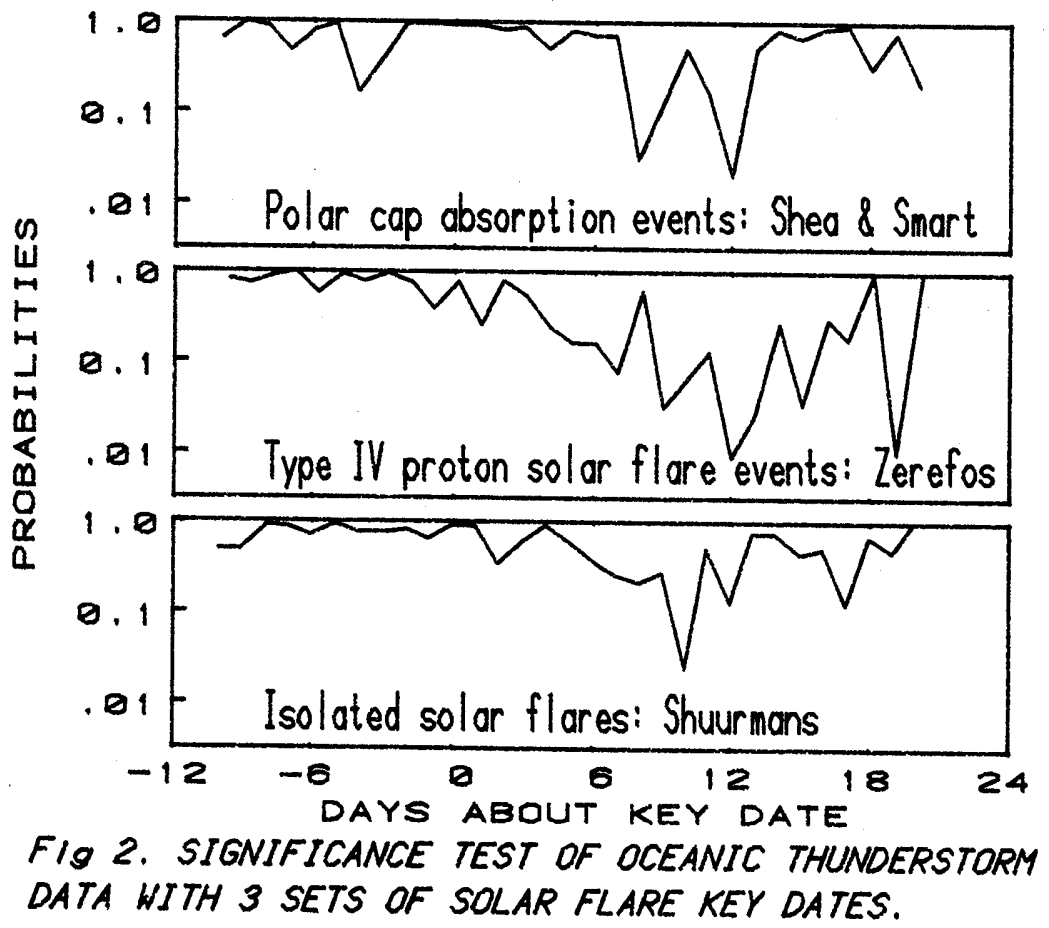




\section{S. Gathman}

This delayed reaction apparently indicates that the variations on oceanic thunderstorms is a secondary effect and is the result of some primary cause at or near the polar caps being propagated through variations in the global weather patterns to the lower latitude zones in which the oceanic thunderstorms were observed.

\section{References.}

Markson, R., Solar modulation of atmospheric electrification and possible implications for the sun - weather relationship, Nature, 273, 103-10.9, 1978.

Lethbridge, M.D。, Cosmic rays and thunderstorm frequency, Geophys. Res. Lett. , 8, 521-522, 1981 .

Trent, E.M. and S.G. Gathman, Oceanic thunderstorms, Pure App1. Geophys., $100,60-69,1972 /$ VIII.

Haurwitz, M.W. and G.W. Brier, A critique of the superposed epoch analysis method: Its application to solar-weather relations, Month. Weath. Rev., $109,2074-2079,1981$.

Schuurmans, C.J.E., The influence of the solar flares on the tropospheric circulation, Netherlands Meteorol. Inst. 98pp, 1969.

Zerefos, C.S., Type IV solar flare proton events, 1956-71, Solarterrestrial physics and meteorology - A working document, Shapley, A。H., H.W. Kroeh1, and J.H. Allen (compilers), SCOSTEP, N.A.S., Washington, 1975.

Shea, M. A。 and D.F。 Smart, Significant solar proton events, 1955-1969, Solar-Terrestrial physics and meteorology - A working document II, Shapley, A.H. and H.W. Kroehl (compilers), SCOSTEP, N.A.S., Washington, 1977.

(Received June 19, 1982; revised September 20, 1982; accepted September 23, 1982) 